\title{
Método de Fio Quente na Determinação das Propriedades Térmicas de Polímeros
}

\author{
Wilson N. dos Santos, Rinaldo Gregorio Filho \\ DEMa, UFSCar
}

\section{Paul Mummery, Andrew Wallwork University of Manchester and UMIST, UK}

\begin{abstract}
Resumo: A técnica de fio quente paralelo normalizada para a determinação da condutividade térmica de materiais cerâmicos foi empregada na determinação das propriedades térmicas de polímeros. As amostras foram preparadas em forma de paralelepípedos retangulares, com dimensões de $(230 \times 80 \times 30) \mathrm{mm}$. Neste trabalho, a condutividade térmica e o calor específico foram simultaneamente determinados a partir do mesmo transiente térmico experimental e a difusividade térmica foi calculada a partir dessas duas propriedades. Cinco diferentes polímeros com diferentes estruturas a temperatura ambiente foram selecionados neste trabalho. Os cálculos foram feitos utilizando-se um método de ajuste por regressão não linear, de tal maneira que todos os pontos experimentais obtidos são considerados nos cálculos dessas propriedades térmicas. $\mathrm{O}$ equipamento utilizado neste trabalho é totalmente automatizado. A reprodutibilidade dos resultados foi muito boa com respeito à condutividade térmica, obtendo-se um desvio máximo de apenas $0,5 \%$ entre os valores máximo e mínimo para todas as amostras ensaiadas, mesmo introduzindo propositadamente alguns defeitos no arranjo experimental, em relação ao modelo teórico. Todavia, pequenos desvios do modelo teórico podem causar drásticas influências nos valores de calor específico, obtendo-se desvios de até $32 \%$ em relação ao arranjo experimental correto. Os resultados experimentais foram então comparados com aqueles encontrados na literatura. As discrepâncias observadas entre alguns desses valores podem estar associadas ao grau de cristalinidade ou à história térmica da amostra, ficando assim mostrada a aplicabilidade desta técnica na determinação das propriedades térmicas de polímeros.
\end{abstract}

Palavras-chave: Polímero, condutividade térmica, calor específico, difusividade térmica, propriedades térmicas, técnica de fio quente.

\section{Hot Wire Technique in the Determination of Thermal Properties of Polymers}

Abstract: The hot wire parallel technique standardized for determining thermal conductivity of ceramic materials was employed in the determination of thermal properties of polymers. Samples were prepared in shape of rectangular parallelepipeds. In this work the thermal conductivity and the specific heat were simultaneously determined from the same experimental thermal transient, and the thermal diffusivity is derived from these properties. Five different polymers with different structures at room temperature were selected and measurements were carried out at room temperature. A non-linear least squares fitting method was employed in the calculations, so that all the experimental points obtained are considered in the thermal properties calculations. The apparatus used in this work is fully automatic. The reproducibility is very good with respect to thermal conductivity, even with a defective experimental arrangement with respect to the theoretical model. However, deviations from the theoretical model causes severe influence on the specific heat values and consequently in the thermal diffusivity. Experimental results were compared with those available in literature, showing the applicability of this technique in the determination of thermal properties of polymers.

Keywords: Polymer, thermal conductivity, specific heat, thermal diffusivity, thermal properties, hot wire technique.

\section{Introdução}

A utilização racional e a economia de energia térmica são importantes demandas de nossa época, não só pela indústria, mas de uma maneira geral. Assim sendo, a transferência de calor com a máxima eficiência possível é tão importante quanto evitar perdas de calor com a utilização de materiais adequados.
As propriedades físicas que determinam a utilização de inúmeros materiais são aquelas propriedades diretamente relacionadas com mudanças de temperatura. Essas propriedades são importantes para todos os materiais, independentemente do seu uso. Entretanto, para aplicações tais como isolantes térmicos, ou sob condições nas quais boa resistência a tensões térmicas é requerida, elas são críticas.

As três propriedades físicas de um material mais impor-

Autor para correspondência: Wilson N. dos Santos, Departamento de Engenharia de Materiais, UFSCar, Via Washington Luiz km 235, Caixa Postal 676, CEP: 13565-905, São Carlos, SP. E-mail:dwns@power.ufscar.br 
tantes do ponto de vista de cálculos térmicos são a condutividade térmica, a difusividade térmica e o calor específico.

Essas três propriedades estão relacionadas entre si pela equação:

$$
\mathrm{a}=\frac{\mathrm{k}}{\rho \mathrm{c}}
$$

onde: $\mathrm{a}=$ difusividade térmica $\left(\mathrm{m}^{2} / \mathrm{s}\right), \mathrm{k}=$ condutividade térmica $(\mathrm{W} / \mathrm{mK}), \rho=$ densidade $\left(\mathrm{kg} / \mathrm{m}^{3}\right)$ e $\mathrm{c}=$ calor específico $(\mathrm{J} / \mathrm{kg} \mathrm{K})$.

A condutividade térmica assume um papel crítico no desempenho de materiais em muitas aplicações. Baixos valores de condutividade térmica são exigidos, quando se pretende minimizar as perdas de calor. Por outro lado, a transferência de calor de uma parte para outra é obtida mais facilmente usando materiais de condutividade térmica mais alta. Essa característica é também desejável, se não crítica, para evitar falhas devido ao choque térmico. Assim sendo, dados confiáveis de condutividade térmica são essenciais na seleção de um material, para que o mesmo possa ter o melhor desempenho possível em uma dada aplicação.

A condutividade térmica é a propriedade que determina os níveis de temperatura de trabalho de um material, e é um importante parâmetro em problemas envolvendo transferência de calor no estado estacionário. Todavia é uma das quantidades físicas cuja medida é muito difícil e requer alta precisão na determinação dos parâmetros envolvidos em seu cálculo.

O calor específico (capacidade térmica por unidade de massa) é também uma propriedade crítica em muitas aplicações. O calor específico, quando se trata de uma amostra pequena, pode ser medido com relativa facilidade. Entretanto, para materiais heterogêneos tendo diferentes fases, onde deve ser medido o calor específico do corpo como um todo, incluindo as diversas fases, e já não é mais possível a preparação de uma amostra pequena e que seja representativa, a medida dessa propriedade torna-se bastante problemática.

A difusividade térmica é uma medida da rapidez com a qual o calor se propaga através de um material. É uma propriedade importante em todos os problemas envolvendo condução de calor no estado não estacionário. Para materiais poliméricos é uma propriedade fundamental no processo de moldagem por injeção, para a determinação do tempo de ciclo de moldagem.

Analogamente ao que ocorre para os materiais cerâmicos, valores confiáveis dessas propriedades térmicas são essenciais para polímeros, tanto em problemas envolvendo o estado estacionário quanto não estacionário. Um exemplo típico de problema envolvendo estado não estacionário, comum em engenharia de polímeros, é o processo de extrusão. Uma vez que durante a extrusão o polímero passa por uma complicada história térmica, o conhecimento de suas propriedades térmicas torna-se crucial na descrição e análise do proces$\mathrm{so}^{[1]}$. Em situações de estado estacionário o conhecimento da condutividade térmica é essencial para a adequada aplicação dos polímeros como isolantes térmicos.

\section{Fundamentos teóricos}

Se um material é isotrópico, a condutividade térmica é a mesma em qualquer direção.

A temperatura $\mathrm{T}$, no instante $\mathrm{t}$ e no ponto $(\mathrm{x}, \mathrm{y}, \mathrm{z})$, num sólido infinito, devido a uma quantidade de calor q que é instantaneamente gerada no tempo $t=0$ no ponto $\left(x^{\prime}, y^{\prime}, z^{\prime}\right)$, é dada pela equação ${ }^{[2]}$ :

$$
\mathrm{T}=\frac{\mathrm{q}}{8 \rho \mathrm{c}(\pi a \mathrm{t})^{3 / 2}} \exp \left\{-\frac{\left(\mathrm{x}-\mathrm{x}^{\prime}\right)^{2}+\left(\mathrm{y}-\mathrm{y}^{\prime}\right)^{2}+\left(\mathrm{z}-\mathrm{z}^{\prime}\right)^{2}}{4 a \mathrm{t}}\right\}
$$

onde: $\rho=$ densidade, $\mathrm{c}=$ calor específico, $\mathrm{a}=\mathrm{k} / \rho \mathrm{c}=$ difusividade térmica.

Para uma fonte linear instantânea de calor, se uma quantidade de calor q'dz é instantaneamente gerada no instante $\mathrm{t}=0$, em todos os pontos sobre uma linha infinita paralela ao eixo $\mathrm{z}$, e passando pelo ponto ( $\left.\mathrm{x}^{\prime}, \mathrm{y}^{\prime}\right)$, a temperatura no ponto $(\mathrm{x}, \mathrm{y})$ no instante t é obtida substituindo-se q na equação 2 por q'dz, e integrando com respeito a z:

$$
\mathrm{T}=\frac{\mathrm{q}^{\prime}}{4 \pi \mathrm{kt}} \exp \left\{-\frac{\left(\mathrm{x}-\mathrm{x}^{\prime}\right)^{2}+\left(\mathrm{y}-\mathrm{y}^{\prime}\right)^{2}}{4 \mathrm{at}}\right\}
$$

sendo q' a quantidade de calor gerada por unidade de comprimento da fonte.

Se uma fonte gera calor a partir do instante $\mathrm{t}=0$, a uma taxa $q^{\prime}(t)$ por unidade de tempo e por unidade de comprimento de uma linha paralela ao eixo z passando pelo ponto ( $\left.\mathrm{x}^{\prime}, \mathrm{y}^{\prime}\right)$, a temperatura no ponto $(\mathrm{x}, \mathrm{y})$ no instante $\mathrm{t}$ é obtida substituindo-se q' na equação 3 por q'( $(\mathrm{t}) \mathrm{dt}$ e integrando com respeito a t:

$$
\mathrm{T}=\frac{\mathrm{q}^{\prime}}{4 \pi \mathrm{k}} \int_{r^{2} / 4 \mathrm{at}}^{\infty} \frac{\mathrm{e}^{-\mathrm{u}}}{\mathrm{u}} \mathrm{du}=\frac{\mathrm{q}^{\prime}}{4 \pi \mathrm{k}}\left[-\mathrm{Ei}\left(-\frac{\mathrm{r}^{2}}{4 \mathrm{at}}\right)\right]
$$

onde $r^{2}=\left(x-x^{\prime}\right)^{2}+\left(y-y^{\prime}\right)^{2}$, e q' é a quantidade de calor gerada por unidade de comprimento da fonte.

A função-Ei(-x), chamada função exponencial integral é definida por:

$$
-\operatorname{Ei}(-x)=\int_{x}^{\infty} \frac{e^{-t}}{t} d t
$$

e pode ser aproximada pela seguinte série de potências:

$$
-\operatorname{Ei}(-x)=\operatorname{E} 1(x)=-\gamma-\ln x-\sum_{n=1}^{\infty} \frac{(-1)^{n} x^{n}}{n n !}
$$

desde que $|\arg \mathrm{x}|<\pi$, e $\arg \mathrm{x}=\arctan \left(\mathrm{x}_{2} / \mathrm{x}_{1}\right)$, sendo $\mathrm{x}=\mathrm{x}_{1}+\mathrm{ix}_{2}$, e $\gamma=0,5772156649 \ldots$ é a constante de Euler.

O método de fio quente é um método direto, absoluto e não estacionário. O método foi descrito em 1888 por Schieirmacher ${ }^{[3]}$. Entretanto, Van Der Held e Van Drunen ${ }^{[4]}$ 
em 1949 foram os que pela primeira vez fizeram uso prático dessa técnica de medida, na determinação da condutividade térmica de líquidos. Haupin ${ }^{[5]} \mathrm{em} 1960$ utilizou pela primeira vez o método do fio quente na determinação da condutividade térmica de materiais cerâmicos e seu trabalho formou a base de todas as variantes do método. No Brasil essa técnica de medida foi utilizada pela primeira vez em 1985 por Santos e Cintra ${ }^{[6]}$, na determinação da condutividade térmica de materiais cerâmicos.

Hoje em dia o método do fio quente é considerado como uma técnica precisa na determinação da condutividade térmica de materiais cerâmicos. Além disso, nessa técnica de medida o conceito de "temperatura média", entre a face quente e a face fria de uma amostra, utilizado nos cálculos pelos métodos calorimétricos é eliminado, uma vez que o cálculo da condutividade é feito a uma temperatura fixa. Nessa técnica, o gradiente de temperatura através da amostra é muito baixo, o que é sem dúvida outra virtude desse método uma vez que um método ideal de medida de condutividade térmica seria aquele capaz de medir essa propriedade segundo um gradiente de temperatura zero através da amostra.

Entretanto, este método tem duas limitações: materiais condutores elétricos e materiais de alta condutividade térmica. No primeiro caso, a solução seria obtida isolando-se eletricamente o fio quente e as amostras. Já para o caso de materiais de alta condutividade térmica, o tempo de registro do transiente térmico torna-se bastante pequeno, comprometendo assim a confiabilidade dos resultados obtidos, a menos que as dimensões das amostras sejam suficientemente grandes, o que por outro lado inviabiliza o processo de medida.

Para uma formulação matemática do método, o fio quente é assumido ser uma fonte de calor ideal (massa $=0$ e, portanto, capacidade térmica $=0$ ), infinitamente longa e fina (diâmetro $=0$ ), a qual é circundada até o infinito pelo material cuja condutividade térmica pretende-se determinar ${ }^{[7]}$. Ao passar uma corrente elétrica constante através do fio, uma quantidade constante de calor, por unidade de tempo e por unidade de comprimento, é liberada pelo fio e vai se propagar através do material. Essa propagação de calor num meio infinito gera, no material, um campo transiente de temperaturas.

Na prática, a fonte teórica linear é aproximada por uma resistência elétrica fina e o sólido infinito é substituído por uma amostra finita. Assim sendo, a capacidade térmica do fio, a resistência de contato entre ele e a amostra e o tamanho finito da amostra são fatores que impõem um tempo mínimo e um tempo máximo a serem utilizados no cálculo da condutividade térmica. A diferença entre as curvas temperatura versus tempo, teórica e real, quando se utiliza um dos quatro possíveis arranjos experimentais desse método é mostrada na Figura 1.

A diferença entre as curvas real e teórica em seu trecho inicial é devido à resistência de contato entre o fio quente e a amostra e à inércia térmica do material, enquanto que a diferença no trecho final é conseqüência do tamanho finito da amostra. A região intermediária, onde as curvas real e teórica exibem o mesmo comportamento, define os limites de tem-

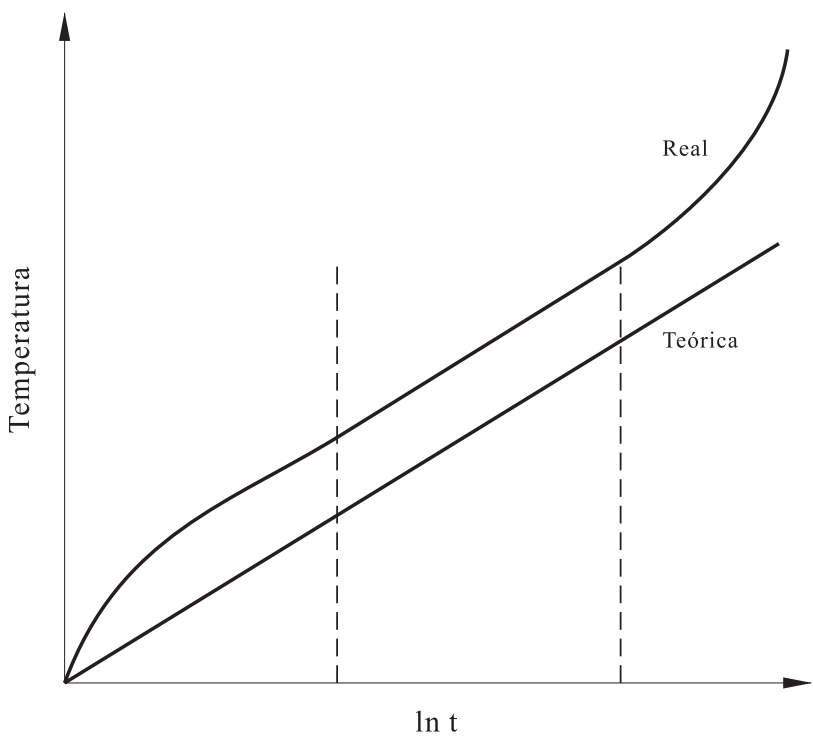

Figura 1. Curvas temperatura versus tempo.

po que devem ser considerados na medida da condutividade térmica pela técnica de fio quente. Assim, o tempo máximo de medida decresce com o aumento da condutividade térmica do material a ser ensaiado. Esse fato implica em uma outra limitação à aplicação deste método para materiais metálicos, que tendo alta condutividade térmica reduziriam drasticamente o tempo máximo de medida.

Alguns cuidados devem ser tomados ao se trabalhar com o método do fio quente para assegurar resultados precisos e consistentes:

1. utilizar uma resistência elétrica fina, que se aproxime o máximo possível da fonte de calor linear teórica;

2. assegurar o melhor contato possível entre ambas as amostras e o fio quente, reduzindo assim os efeitos da resistência de contato;

3. não considerar, para efeito de cálculo, o trecho inicial da curva, eliminando assim os efeitos de contato térmico entre a resistência elétrica e o material da amostra;

4. limitar o tempo de ensaio para assegurar que o tamanho finito da amostra não afete as temperaturas medidas.

Hoje são conhecidas quatro variações do método de fio quente: técnica padrão, técnica em paralelo, técnica de resistência e técnica de dois termopares. O modelo teórico é o mesmo, sendo que a diferença básica entre essas variações está no procedimento de medida da temperatura. Conseqüentemente, a equação final obtida para o cálculo da condutividade térmica é diferente para cada uma dessas variações. Em todas elas, entretanto, são sempre necessários dois corpos-de-prova. Neste trabalho foi utilizada a técnica de fio quente paralelo.

Nesta técnica são necessárias duas amostras em forma de paralelepípedos retangulares ou de semicilindros. Em um dos corpos de prova são feitas duas ranhuras paralelas: por uma delas passa o fio quente e pela outra um termopar. A profundidade desses sulcos deve corresponder aproximadamente ao diâmetro dos fios a serem neles inseridos, e a dis- 


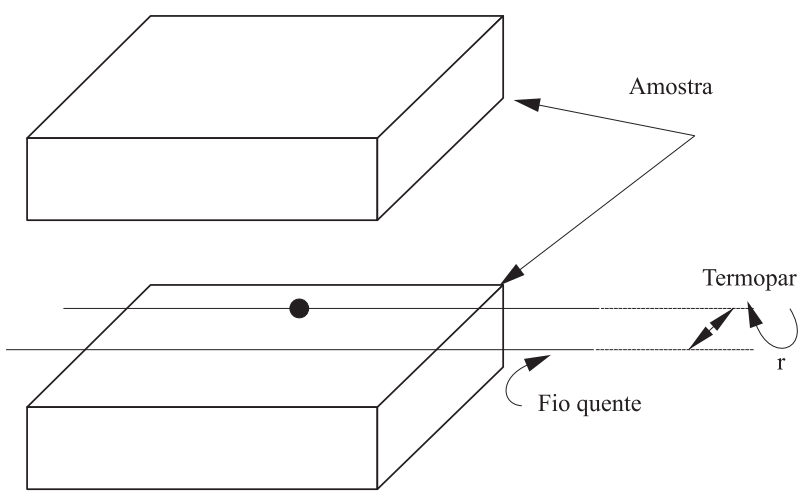

Figura 2. Esquema do arranjo dos corpos-de-prova na técnica de fio quente paralelo.

tância recomendada entre eles é de 15 a $17 \mathrm{~mm}$. Sobre esse corpo de prova é então colocado o segundo corpo. As superfícies em contato devem ser bem polidas para proporcionar o melhor contato térmico possível. A junção de referência do termopar é mantida a uma temperatura constante (referência). A Figura 2 mostra um diagrama esquemático do arranjo experimental dos corpos de prova.

No processo de medida da condutividade térmica, faz-se passar uma corrente elétrica constante através do fio, e registra-se o aumento de temperatura no material a uma distância $r$ desse fio, a partir da temperatura inicial de equilíbrio. No caso de medida em temperaturas superiores a ambiente, os corpos de prova e o dispositivo de medida são colocados no interior de um forno. A condutividade térmica é calculada de acordo com a equação 4 , que combinada com a equação 1 pode ser escrita na forma:

$$
\mathrm{k}=\frac{-\mathrm{q}^{\prime}}{4 \pi \mathrm{T}} \operatorname{Ei}\left(-\frac{\rho c r^{2}}{4 k t}\right)
$$

onde: q' $=$ densidade linear de potência $(\mathrm{W} / \mathrm{m}), \mathrm{T}=$ excesso de temperatura em relação à temperatura inicial de referência $(K), r=$ distância radial a partir do fio quente $(\mathrm{m}), \mathrm{t}=$ tempo, contado a partir do início da liberação de calor (s), isto é, instante em que o circuito de aquecimento é ligado e -Ei = função exponencial integral, definida pela equação 5 .

Essa variante da técnica de fio quente foi normalizada para materiais cerâmicos em 1978 pela norma DIN-51046Parte 2. De acordo com essa norma a condutividade térmica é determinada para vários pares selecionados de tempos te $2 t$ usando-se a equação 7 e em seguida é feita uma média aritmética entre os valores obtidos. Neste trabalho é utilizado o procedimento de cálculo introduzido em 1986 por Santos e Cintra ${ }^{[6]}$, no qual é feito um duplo ajuste por regressão não linear, obtendo-se simultaneamente a partir do mesmo transiente térmico experimental a condutividade térmica, o calor específico e a difusividade térmica do material. Nesse procedimento de cálculo, utilizando-se a equação 7 , a condutividade térmica e o calor específicos são ajustados de modo a se obter a melhor aproximação possível entre o transiente de temperatura registrado experimentalmente e aquele previsto pelo modelo teórico. Nesse caso as

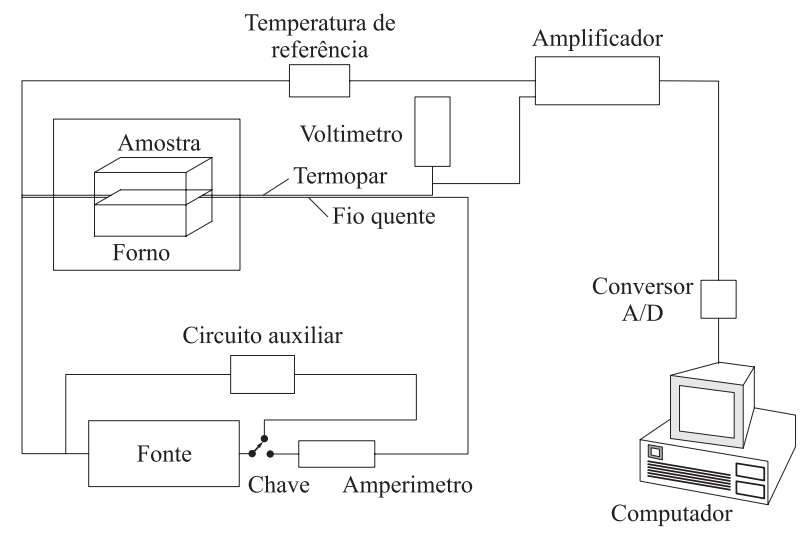

Figura 3. Diagrama esquemático do arranjo experimental utilizado.

duas propriedades térmicas, condutividade térmica e calor específico são determinadas simultaneamente a partir do mesmo transiente térmico. Conhecendo-se então a densidade do material, a difusividade térmica pode ser então calculada pela equação 1 . O sistema de aquisição e processamento de dados utilizado neste trabalho é totalmente automatizado: o transiente de temperatura que é detectado pelo termopar é processado em um microcomputador, via um conversor analógico-digital, usando um programa computacional especialmente desenvolvido para esse objetivo. A Figura 3 mostra o arranjo experimental utilizado no Laboratório de Propriedades Térmicas do DEMa/UFSCar.

\section{Preparação das amostras}

As amostras em forma de paralelepípedos retangulares, com dimensões de (230x80x30)mm, foram obtidas a partir de placas de polímeros comerciais. Os sulcos paralelos foram feitos aplicando-se uma corrente elétrica adequada no próprio fio quente e no termopar e pressionando-os contra a superfície da mostra. A fusão local e posterior resfriamento do polímero providenciaram um contato perfeito entre o fio e a amostra. Em todas as amostras a distância r entre o fio quente e o termopar foi mantida entre 15 e $16 \mathrm{~mm}$. Para assegurar o melhor contato possível entre as amostras, essas foram pressionadas uma contra a outra, utilizando-se anéis de aço inoxidável. Com o objetivo de minimizar o ruído eletrônico e eliminar flutuações do amplificador causadas por tensões muito baixas, a junção de referência foi mantida a $0{ }^{\circ} \mathrm{C}$. Esse procedimento é recomendado, uma vez que deve ser baixa a densidade linear de potência aplicada ao fio quente para evitar regiões de fusão e degradação no interior da amostra, tendo-se em mente o baixo ponto de fusão ou amolecimento dos polímeros, quando comparado com materiais cerâmicos. Foram preparadas 5 amostras de diferentes polímeros: nylon 66, polipropileno (PP), poli(metacrilato de metila) (PMMA), poli(cloreto de vinila) (PVC) e poliuretano (PU).

\section{Resultados e Discussão}

As medidas experimentais foram feitas a temperatura ambiente. A condutividade térmica e o calor específico foram determinados a partir do transiente de temperatura correspondente a cada amostra, utilizando-se para isso um método de 
Tabela 1. Resultados obtidos pela técnica do fio quente paralelo e da literatura.

\begin{tabular}{|c|c|c|c|c|c|c|}
\hline \multirow[b]{2}{*}{ Polímero } & \multicolumn{3}{|c|}{ Fio Quente Paralelo } & \multicolumn{3}{|c|}{ Literatura } \\
\hline & $\begin{array}{c}\mathbf{k} \\
(\mathbf{W} / \mathbf{m K})\end{array}$ & $\begin{array}{c}c \\
(J / k g K)\end{array}$ & $\begin{array}{c}a \\
\left(\mathbf{x} 10^{-7} \mathrm{~m}^{2} / \mathrm{s}\right)\end{array}$ & $\begin{array}{c}\mathbf{k} \\
(\mathbf{W} / \mathbf{m K})\end{array}$ & $\underset{(J / k g K)}{c}$ & $\begin{array}{c}\mathbf{a} \\
\left(\mathbf{x} 10^{-7} \mathrm{~m}^{2} / \mathrm{s}\right)\end{array}$ \\
\hline \multirow{3}{*}{ Nylon 66} & \multirow{3}{*}{0,3022} & \multirow{3}{*}{1783,66} & \multirow{3}{*}{1,4730} & $0,2400^{(8)}$ & $1700,00^{(8)}$ & $1,01^{(8)}$ \\
\hline & & & & $0,3000^{(9)}$ & $1674,40^{(10)}$ & $1,30^{(11)}$ \\
\hline & & & & $0,2400^{(10)}$ & & \\
\hline \multirow{3}{*}{ PP } & \multirow{3}{*}{0,2321} & \multirow{3}{*}{1812,98} & \multirow{3}{*}{1,4550} & $0,2400(8)$ & $2100,00^{(1)}$ & $0,6500^{(8)}$ \\
\hline & & & & & $1925,56^{(8)}$ & $0,9000^{(11)}$ \\
\hline & & & & & $1925,56^{(10)}$ & \\
\hline \multirow{3}{*}{ PMMA } & \multirow{3}{*}{0,1955} & \multirow{3}{*}{1422,41} & \multirow{3}{*}{1,1550} & $0,2000^{(1)}$ & $1450,00^{(1)}$ & $1,09^{(8)}$ \\
\hline & & & & $0,2000^{(8)}$ & $1465,10^{(8)}$ & \\
\hline & & & & $0,2100^{(10)}$ & $1465,10^{(10)}$ & \\
\hline \multirow{5}{*}{$\begin{array}{l}\text { PVC } \\
\text { rígido }\end{array}$} & \multirow{5}{*}{0,1858} & \multirow{5}{*}{997,70} & \multirow{5}{*}{1,3590} & $0,1600^{(8)}$ & $1004,64^{(8)}$ & $1,16^{(8)}$ \\
\hline & & & & $0,1300^{(1,10)}$ & $837,0^{(1,10)}$ & $1,10^{(11)}$ \\
\hline & & & & & & \\
\hline & & & & $0,2900^{(10)}$ & $1172,08^{(10)}$ & \\
\hline & & & & $0,2100^{(1)}$ & $1100,0^{(1)}$ & \\
\hline PU espuma & 0,0305 & 1447,79 & 6,5740 & $0,032(8)$ & - & - \\
\hline
\end{tabular}

análise por regressão não linear ${ }^{[6]}$. A difusividade térmica foi então calculada a partir dos valores dessas duas propriedades e da densidade da amostra, utilizando-se para isso a equação 1. Os resultados experimentais obtidos são comparados com aqueles encontrados na literatura e mostrados na Tabela 1.

Os resultados mostrados na Tabela 1 permitem afirmar que o método de fio quente paralelo é uma técnica adequada na determinação das propriedades térmicas de polímeros, embora alguns desvios possam ser encontrados quando alguns resultados são comparados com os correspondentes valores encontrados na literatura. Talvez as discrepâncias possam ser associadas ao grau de cristalinidade ou a história térmica da amostra, uma vez que esses fatores podem afetar as propriedade térmicas de polímeros.

O procedimento de análise por regressão adotado neste trabalho é uma ferramenta matemática muito útil, permitindo a determinação simultânea da condutividade térmica, calor específico e difusividade térmica. Essa é a grande vantagem desta técnica. A qualidade do ajuste pode ser demonstrada pelo coeficiente de correlação. Todos os coeficientes obtidos foram maiores que 0,998 . Para o ajuste perfeito esse coeficiente seria igual a 1 .

\section{Influência do fluxo de calor}

Os valores das propriedades térmicas não devem depender da densidade linear de potência aplicada ao fio quente.
Se esse fenômeno ocorrer, ele só pode ser atribuído a perturbações no método. Para o PMMA, escolhido aleatoriamente, três diferentes valores de densidade linear de potência foram aplicadas na determinação dessas propriedades: 25, 40 e 60 $\mathrm{W} / \mathrm{m}$. Os desvios máximos entre as condutividades térmicas, calores específicos e difusividades térmicas calculados com essas três densidades lineares de potência foram respectivamente $1,46 \%, 1,17 \%$ e $2,61 \%$, mostrando assim, como era esperado, que o fluxo de calor não influi nos valores dos resultados experimentais obtidos. Todavia deve-se ter sempre em mente que, quanto mais baixa for a densidade linear de potência aplicada, maior será o nível de ruído detectado no transiente de temperatura.

\section{Reprodutibilidade}

A reprodutibilidade das medidas é muito boa desde que os aspectos referentes ao modelo teórico sejam observados cuidadosamente. A Tabela 2 mostra os desvios percentuais em relação ao valor médio. Com relação à condutividade térmica, a reprodutibilidade ainda é muito boa mesmo com um arranjo experimental defeituoso em relação ao modelo teórico. Todavia, desvios do modelo teórico influenciam drasticamente os valores de calor específico e consequentemente da difusividade térmica. Com um arranjo propositadamente defeituoso, no que se refere ao embutimento do termopar, obteve-se um desvio de apenas $0,5 \%$ na condutividade tér- 
Tabela 2. Desvios percentuais em relação ao valor médio.

\begin{tabular}{|c|c|c|c|c|c|c|}
\hline \multirow{2}{*}{ Polímero } & \multicolumn{2}{|c|}{ Valores Medidos } & \multicolumn{2}{|c|}{ Valor Médio } & \multicolumn{2}{|c|}{$\delta$} \\
\hline & $\begin{array}{c}\mathbf{k} \\
(\mathbf{W} / \mathbf{m K})\end{array}$ & $\begin{array}{c}c \\
(J / k g K)\end{array}$ & $\begin{array}{c}\mathbf{k} \\
(\mathbf{W} / \mathbf{m K})\end{array}$ & $\underset{(\mathrm{J} / \mathrm{kgK})}{\mathrm{c}}$ & $\begin{array}{c}\delta_{k} \\
(\%)\end{array}$ & $\begin{array}{l}\delta_{c} \\
(\%)\end{array}$ \\
\hline \multirow{3}{*}{ Nylon 66} & 0,3015 & 1770,32 & \multirow{3}{*}{0,3022} & \multirow{3}{*}{1783.66} & 0,23 & 0,75 \\
\hline & 0,3028 & 1793,55 & & & 0,20 & 0,55 \\
\hline & 0,3023 & 1787,11 & & & 0,03 & 0,19 \\
\hline \multirow{3}{*}{ PP } & 0,2330 & 1791,01 & \multirow{3}{*}{0,2321} & \multirow{3}{*}{1812,98} & 0,39 & 1,23 \\
\hline & 0,2317 & 1830,76 & & & 0,17 & 0,98 \\
\hline & 0,2316 & 1817,17 & & & 0,22 & 0,23 \\
\hline \multirow{3}{*}{ PMMA } & 0,1960 & 1433,91 & \multirow{3}{*}{0,1955} & \multirow{3}{*}{1422,41} & 0,26 & 0,81 \\
\hline & 0,1957 & 1410,78 & & & 0,10 & 0,82 \\
\hline & 0,1948 & 1422,54 & & & 0,36 & 0,01 \\
\hline \multirow{3}{*}{ PVC rígido } & 0,1865 & 1000,99 & \multirow{3}{*}{0,1858} & \multirow{3}{*}{997,70} & 0,38 & 0,33 \\
\hline & 0,1857 & 992,82 & & & 0,05 & 0,49 \\
\hline & 0,1852 & 999,28 & & & 0,32 & 0,16 \\
\hline \multirow{3}{*}{ PU espuma } & 0,0304 & 1460,79 & \multirow{3}{*}{0,0305} & \multirow{3}{*}{1447,79} & 0,33 & 0,90 \\
\hline & 0,0306 & 1443,87 & & & 0,33 & 0,27 \\
\hline & 0,0305 & 1438,71 & & & 0,00 & 0,63 \\
\hline
\end{tabular}

$\delta=$ Desvio percentual em relação ao valor médio

mica e de $32 \%$ no calor específico, em relação ao arranjo experimental correto. Esse resultado mostra a importância da observância das hipóteses previstas pelo modelo teórico.

\section{Conclusões}

A técnica de fio quente, muito utilizada na determinação das propriedades térmicas de materiais cerâmicos, mostrouse extremamente adequada também para materiais poliméricos, desde que seja possível a preparação de amostras com as dimensões previstas pelo modelo teórico. A grande vantagem dessa técnica é a utilização do procedimento de análise por regressão não linear adotado neste trabalho. Essa é uma ferramenta matemática muito útil, permitindo a determinação simultânea da condutividade térmica, calor específico e difusividade térmica do material.

\section{Agradecimentos}

Os autores agradecem à FAPESP (Proc. 2001/08225-4) e ao CNPq (Proc. 302969/2002-0 e Proc. 301452/1991-1) pelo apoio financeiro recebido.

\section{Referências Bibliográficas}

1. Rauwendaal, C. -"Polymer Extrusion", Hanser Publishers, Carl Hanser Verlag, Munich (1986).
2. Carslaw, H. S.; Jaeger, J. C. - "Conduction of Heat in Solids", Oxford University Press, Oxford (1959).

3. Schieirmacher, A. L. - Wiedemann Ann Phys, 34, p.38 (1888).

4. Van Der Held, E. F. M. \& Van Drunen, F. G. - Physics, 15, 10, p.865 (1949).

5. Haupin, W.E. - Am Ceram Soc Bull, 39, 3, p.139 (1960).

6. Santos, W. N. \& Cintra Filho, J. S. - Cerâmica, 32, 198, p.151 (1986).

7. Boer, J.; Butter, J.; Grosskopf, B. \& Jeschke, P. Refractories Journal, 55, p.22 (1980).

8. Crawford, R. J. -"Plastics Engineering", ButterworthHeinemann, Oxford, (1998).

9. Handbook of Chemistry and Physics, CRC Press, New York, (1996).

10. Encyclopedia of Polymer Science and Technology, Interscience Publishers, Vol. 13, John Wiley \& Sons Inc., New York, (1970).

11. Ogorkiewicz, R. M. - "Thermoplastics-Properties and Design”, John Wiley \& Sons, London, (1974).

Enviado: $14 / 07 / 04$

Reenviado: 05/10/04 Aprovado: 09/10/04 